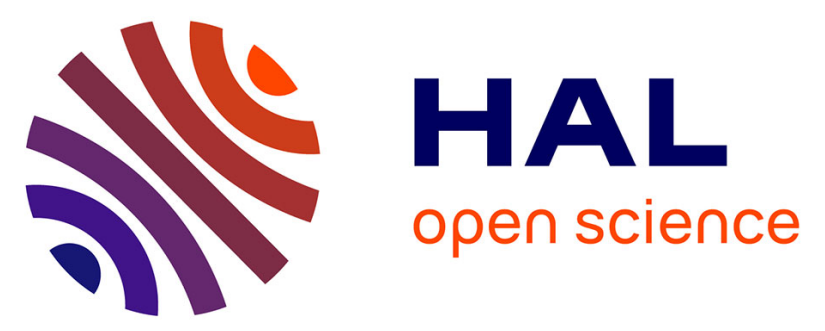

\title{
Outil de sensibilisation des conducteurs âgés aux capacités requises pour une conduite automobile sécuritaire et responsable (OSCAR) : développement et validation
}

Mélanie Levasseur, Thérèse Audet, Isabelle Gélinas, Michel Bédard, Judith

Renaud, Jean-claude Coallier, Marie-ève Langlais, France-hélène Therrien

\section{To cite this version:}

Mélanie Levasseur, Thérèse Audet, Isabelle Gélinas, Michel Bédard, Judith Renaud, et al.. Outil de sensibilisation des conducteurs âgés aux capacités requises pour une conduite automobile sécuritaire et responsable (OSCAR) : développement et validation. RTS - Recherche Transports Sécurité, 2015, Conducteurs âgés, 2014 (04), pp.257-269. 10.4074/S076189801400404X . hal-01670607

\section{HAL Id: hal-01670607 https://hal.science/hal-01670607}

Submitted on 21 Dec 2017

HAL is a multi-disciplinary open access archive for the deposit and dissemination of scientific research documents, whether they are published or not. The documents may come from teaching and research institutions in France or abroad, or from public or private research centers.
L'archive ouverte pluridisciplinaire HAL, est destinée au dépôt et à la diffusion de documents scientifiques de niveau recherche, publiés ou non, émanant des établissements d'enseignement et de recherche français ou étrangers, des laboratoires publics ou privés. 


\title{
Outil de sensibilisation des conducteurs âgés aux capacités requises pour une conduite automobile sécuritaire et responsable (OSCAR) : développement et validation
}

\author{
Awareness tool for safe and responsible driving (OSCAR): \\ development and validation
}

\author{
Mélanie Levasseur - Thérèse Audet · Isabelle Gélinas · Michel Bédard · Judith Renaud · Jean-Claude Coallier . \\ Marie-Ève Langlais · France-Hélène Therrien
}

(C) IFSTTAR et Éditions NecPlus 2014

\author{
Mélanie Levasseur $(\bowtie)$ \\ Université de Sherbrooke, Centre de recherche sur le vieillissement 1036, \\ rue Belvédère Sud Sherbrooke QC J1H 4C4, Canada CSSS-IUGS \\ e-mail : Melanie.Levasseur@USherbrooke.ca \\ Thérèse Audet $(\triangle)$ \\ Université de Sherbrooke, Centre de recherche sur le vieillissement, \\ SSSS-IUGS \\ 1036, rue Belvédère Sud \\ Sherbrooke QC J1H 4C4, Canada \\ e-mail : therese.audet@usherbrooke.ca \\ Isabelle Gélinas $(\bowtie)$ \\ McGill University, Centre de recherche interdisciplinaire en réadaptation \\ 3654 prom Sir-William-Osler \\ Montréal, QC, Canada H3G 1Y5 \\ e-mail : isabelle.gelinas@mcgill.ca
}

Michel Bédard $(\bowtie)$

Lakehead University, Centre for Research on Safe Driving

955 Oliver Road

Thunder Bay, ON P7B 5E1 Canada

e-mail : isabelle.gelinas@mcgill.ca

Judith Renaud $(\bowtie)$

Université de Montréal, École d'optométrie

3744 Jean-Brillant

Montréal, Qc H3T 1P1

e-mail : judith.renaud@umontreal.ca

Jean-Claude Coallier $(\bowtie)$

Université de Sherbrooke, Centre de recherche sur le vieillissement,

SSSS-IUGS

1036, rue Belvédère Sud

Sherbrooke QC J1H 4C4, Canada

e-mail : jean-claude.coaillier@usherbrooke.ca

Marie-Ève Langlais $(\bowtie)$

Université de Sherbrooke, Centre de réadaptation Estrie

300, rue King Est, bureau 200

Sherbrooke (Québec) J1G 1B1

e-mail : melanglais.cre@ssss.gouv.qc.ca

France-Hélène Therrien $(\bowtie)$

Université de Sherbrooke, Centre de recherche sur le vieillissement,

SSSS-IUGS

1036, rue Belvédère Sud

Sherbrooke QC J1H 4C4, Canada

e-mail : france-helene.therrien@usherbrooke.ca
Résumé L'objectif de cette étude était de développer et de valider un outil d'intervention pour sensibiliser les conducteurs âgés aux capacités requises et aux stratégies compensatoires pour une conduite automobile sécuritaire. Une recension élargie des écrits a d'abord été réalisée pour développer l'Outil de Sensibilisation des conducteurs âgés aux capacités requises et aux stratégies compensatoires pour une conduite automobile sécuritaire et responsable (OSCAR). L'outil comprend une série de 15 questions et 15 capsules éducatives en lien avec le vieillissement et la conduite automobile. Un dispositif expérimental incluant un pré-test et un post-test huit à dix semaines après l'intervention a ensuite été utilisé auprès de 48 personnes âgées entre 67 et 84 ans qui conduisaient au moins une fois par semaine. À la suite de la passation de l'OSCAR, les résultats démontrent que l'intérêt, l'ouverture et les connaissances des aînés sur les capacités requises et les stratégies compensatoires ont augmenté de façon significative $(p<0,01)$. La majorité des participants ont confirmé que des changements se sont produits dans au moins une de leurs capacités. En outre, toujours à la suite de l'OSCAR, la moitié des conducteurs âgés ont commencé à utiliser plus de cinq stratégies compensatoires. Tout en faisant la promotion d'une conduite automobile sécuritaire, cette intervention pourrait aider les personnes âgées à maintenir ou à accroitre leur mobilité lors des déplacements. D'autres études sont nécessaires pour évaluer l'efficacité et l'implantation de l'OSCAR.

Mots clés conducteurs âgés · mobilité · outil de sensibilisation $\cdot$ conduite automobile $\cdot$ stratégies compensatoires $\cdot$ OSCAR

Abstract The objective of the study was to develop and validate an intervention tool to sensitize older drivers to safe driving. A scoping study was first conducted 
to develop an awareness tool for safe and responsible automobile driving (OSCAR). The tool includes a series of 15 questions and 15 educational capsules on aging and driving. A pre-experimental design, including a pre-test and post-test conducted eight to ten weeks after exposure to the intervention, was used with 48 drivers aged between 67 and 84 who drove at least once a week. Overall, the results demonstrate that OSCAR increased the older drivers' interest, openness and knowledge about the abilities required and compensatory strategies for driving $(p<0.01)$. After exposure to OSCAR, the majority of the participants confirmed that changes occurred in at least one of their abilities. Moreover, half of the older drivers started using more than five compensatory strategies. In summary, in addition to increasing older adults' interest, openness and knowledge to discussion about driving, OSCAR also improved awareness of the changes that could negatively impact safe driving and enhanced utilization of compensatory strategies. While promoting safe driving and the prevention of crashes and injuries, this intervention could ultimately help older adults maintain or increase their transportation mobility. More studies are needed to further evaluate OSCAR and improve its effectiveness.

Keywords Older drivers · Mobility · Awareness tool - Safe driving $\cdot$ Compensatory strategies $\cdot$ OSCAR

\section{Introduction}

La conduite automobile est une activité importante pour l'autonomie et le bien-être des personnes âgées [1-5], et représente souvent leur principal moyen de transport $[2$, 4]. Considérant ce moyen privilégié et le vieillissement de la population, le nombre de permis de conduire délivrés aux conducteurs âgés a plus que doublé dans les dix dernières années et l'âge moyen des conducteurs ne cesse d'augmenter [4, 7]. Toutefois, étant une tâche complexe, la conduite requiert une utilisation efficiente et adéquate des capacités motrices, sensorielles, et cognitives [4], dont l'intégrité est influencée par le vieillissement normal et pathologique. Spécifiquement, les meilleurs prédicteurs du risque de collision des personnes âgées seraient les déficits d'ordre visuo-perceptuel, attentionnel et cognitif [4].

Une diminution des capacités de conduite peut entraver la sécurité du conducteur âgé et des autres utilisateurs des voies publiques $[8,9]$. Pour un nombre équivalent de kilomètres parcourus, les conducteurs âgés ont un taux de collision plus élevé que la population générale [4, 8-11]. En raison d'une fragilité accrue [4, 12], les aînés présentent un taux plus élevé de mortalité et de morbidité lors de collisions de la route que les adultes plus jeunes $[2,5$, 10]. Ces collisions sont d'ailleurs la $1^{\text {re }}$ cause de décès par blessures non intentionnelles chez les personnes de 65 à 74 ans $[9,11]$, et la $2^{\mathrm{e}}$ chez les 75 à 84 ans [13]. Puisque bien connaitre un risque contribue à le diminuer, il importe que l'individu ait de bonnes connaissances des risques associés à une diminution des capacités à conduire un véhicule.

Identifiée comme une méthode d'intervention privilégiée pour promouvoir et améliorer la santé individuelle et collective $[14,15]$, la sensibilisation peut favoriser une conduite sécuritaire chez les conducteurs âgés [9]. La sensibilisation permet au conducteur âgé de comprendre davantage l'influence de ses propres déficits sur ses capacités à conduire un véhicule et, lorsque pertinent, de percevoir un besoin de changement et de présenter une ouverture quant à l'utilisation de stratégies compensatoires. Les stratégies compensatoires sont des moyens conscients utilisés par une personne pour pallier à la diminution de ses capacités [16], par exemple, éviter de conduire à la noirceur parce que l'éblouissement causé par les phares des autres voitures devient inconfortable ou choisir un véhicule plus facile à conduire (transmission automatique, options électriques, etc.) lorsque certains problèmes musculo-squelettiques (ex. : douleurs articulaires) surviennent. Plus la sensibilisation est réalisée tôt, plus l'adaptation et la réadaptation sont facilitées [7], et le changement simplifié [17]. Cependant, peu d'outils de sensibilisation (voir section résultats) ont été évalués rigoureusement et sont disponibles en français. Par conséquent, le développement, l'adaptation au contexte québécois et la validation d'un outil de sensibilisation fondé sur les données probantes et l'opinion d'experts sont requis. L'objectif de la présente recherche était donc de développer et de valider un outil d'intervention pour sensibiliser les conducteurs âgés aux capacités requises et aux stratégies compensatoires en conduite automobile.

\section{Méthodologie}

\section{Développement de l'outil}

Pour atteindre ces objectifs et être cohérents aux lignes directrices guidant le développement d'outils [18], un schéma méthodologique rigoureux et précis (Figure 1) a été utilisé. Une recension élargie (scoping study) des écrits scientifiques et de la littérature grise de 1980 à août $2010 \mathrm{a}$ d'abord été réalisée pour inventorier les outils en lien avec la conduite automobile et la sensibilisation des aînés. Huit bases de données (Medline, OTDBASE, CINAHL, AMED, MANTIS, Embase, TRIS et RITA) ont été questionnées en combinant les mots-clés selon la stratégie suivante : 1. driving or driver*; ET 2. aging, ageing, older, elderly ou aged ; ET 3. self-assess*/evaluate*; ET 4) questionnaire, tool ou test. Afin de sélectionner le meilleur, les outils de sensibilisation ou de dépistage recensés ont ensuite été évalués en fonction de 15 critères prédéfinis (Figure 1), puis discutés 


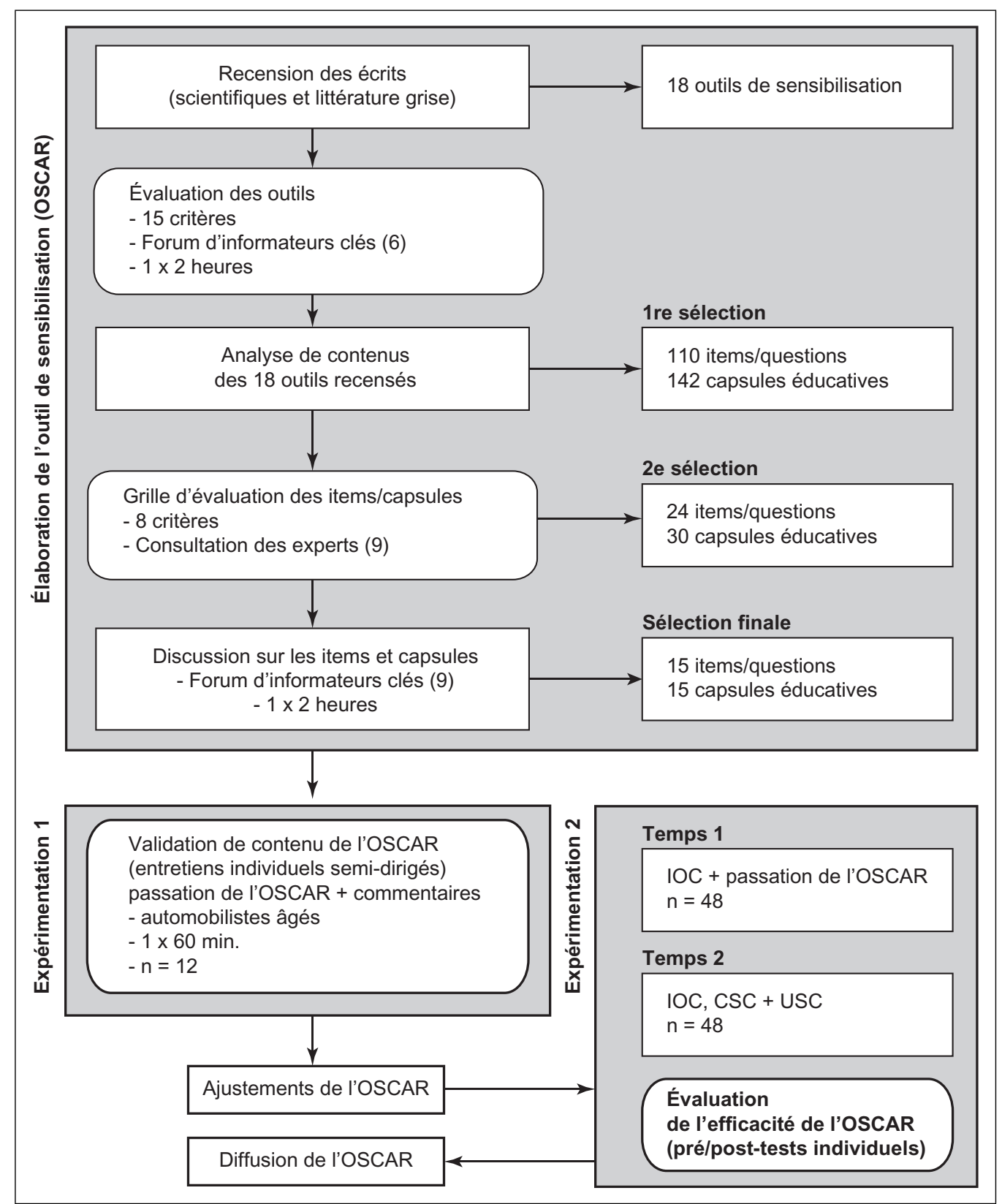

Fig. 1 Schéma méthodologique sous-jacent au développement de l'outil

lors d'un premier forum d'informateurs-clés $(\mathrm{n}=6)$, c'està-dire un groupe d'experts avec discussion et adoption de propositions par consensus. Par la suite, une analyse de contenu utilisant le modèle de Michon [19] a permis de décortiquer les outils en classifiant les capacités requises pour une conduite automobile sécuritaire selon les caractéristiques propres aux tâches impliquées, et de situer, principalement au niveau stratégique, l'intervention de sensibilisation et l'utilisation de stratégies compensatoires, et de procéder à une première sélection d'items et de capsules éducatives. Le modèle de Michon décrit la conduite automobile à partir de trois niveaux distincts de traitement de l'information
[19] : 1. stratégique, c'est-à-dire relatif à la planification de l'itinéraire, ce qui comprend la modification du trajet, la prise en considération de ses erreurs et la présence d'un comportement adéquat; ce niveau nécessite une analyse de plusieurs minutes et intègre les stratégies compensatoires, 2. tactique, c'est-à-dire qui regroupe différentes actions, nécessitant quelques secondes seulement de réflexion, telles que les changements de voies, l'évitement d'obstacles, le choix de la distance entre les véhicules et le choix de la vitesse, et 3. opérationnel, c'est-à-dire de la mise en œuvre, en millisecondes, des différentes manœuvres en lien avec les niveaux supérieurs tels accélération, freinage, contrôle 
du volant et repérage de la signalisation. Afin de poursuivre la sélection, les items et capsules retenus ont ensuite été soumis à neuf experts pour une évaluation en fonction de huit critères prédéfinis (Figure 1). Lors de ce second forum, les meilleurs items et capsules éducatives ont été discutés pour arriver à un consensus sur une sélection finale et une première version de l'outil.

\section{Expérimentation 1 : validation du contenu de l'outil auprès de 12 aînés}

Une expérimentation initiale de l'outil a été réalisée afin de valider son contenu auprès de 12 conducteurs âgés de 65 ans et plus, conduisant au moins une fois par semaine et sans déficit cognitif. Le recrutement de ces automobilistes a été réalisé grâce à un échantillonnage en boule de neige et par le biais d'affiches dans différentes organisations d'aînés. Une rencontre individuelle de chaque participant a subséquemment permis de remplir un questionnaire d'information sociodémographique, de prendre connaissance de l'outil développé, puis de réaliser un entretien semi-dirigé permettant de recueillir leurs commentaires et perceptions en lien avec l'outil. Le guide d'entretien comportait 17 questions ouvertes en lien, notamment, avec l'impact de l'outil sur la prise de conscience des changements survenus et sur l'intention d'adopter certaines stratégies compensatoires, l'utilité de l'outil globalement et spécifiquement comme aide à la discussion, et la mise en page de l'outil.

\section{Expérimentation 2 : exploration des effets de l'outil auprès de 48 aînés}

Enfin, une deuxième expérimentation a permis de vérifier l'efficacité de l'outil grâce à un dispositif pré-expérimental incluant un pré-test avant de débuter l'intervention $\left(\mathrm{T}_{0}\right)$ et un post-test huit à dix semaines après l'intervention $\left(T_{1}\right)$. Lors de l'intervention, les participants étaient invités à lire l'outil en prenant autant de temps qu'il le souhaitait. Toutefois, afin de reproduire des conditions reflétant une exposition minimale, les participants ne pouvaient conserver l'outil et étaient réévalués 8 à 10 semaines après en avoir pris connaissance. Cette deuxième expérimentation a été réalisée auprès de 48 conducteurs âgés, ce qui permettait de détecter une taille de l'effet modéré de 0,42 en fonction d'un test t bilatéral pour données appariées, à un niveau alpha fixé à 0,05 et une puissance de $80 \%$ [20]. Les participants devaient avoir de bonnes capacités cognitives, communiquer en français et conduire au moins une fois par semaine. Ils ont été recrutés selon une stratégie de convenance jusqu'à l'atteinte de la taille d'échantillon désirée et à partir d'une liste des personnes ayant participé antérieurement, et de dépliants distribués dans la communauté et les médias locaux. Trois questionnaires développés pour les besoins de l'étude ont été utilisés pour recueillir les données : 1 . Intérêt, ouverture et connaissances (IOC), 2. Changements survenus dans les capacités (CSC), et 3. Utilisation de stratégies compensatoires (USC). Le premier questionnaire (IOC) a été complété à $\mathrm{T}_{0}$ et $\mathrm{T}_{1}$, alors que les deux derniers (CSC et USC) l'ont été à $\mathrm{T}_{1}$ uniquement (Figure 1). L'IOC contient 27 questions à choix multiples dont la majorité présente quatre ou cinq options dont une seule représente la bonne réponse. Ce questionnaire inclut trois sections (nombre de questions) : (i) intérêt envers l'information sur la conduite automobile et l'ouverture à discuter de ses capacités de conduite et de l'utilisation de stratégies compensatoires (5 questions, par exemple : « Êtes-vous intéressé par des reportages télévisés, radiophoniques, ou des articles de journaux qui parlent des capacités à conduire des personnes âgées ? »; score variant entre 0-19) ; (ii) connaissances sur la sécurité routière et les capacités nécessaires à une conduite sécuritaire ( 9 questions, par exemple : « À quelle fréquence devriez-vous faire évaluer votre vision ? »; score variant entre 0-9) ; et (iii) connaissances sur l'impact du vieillissement sur les capacités et sur les stratégies compensatoires (13 questions, par exemple : « De quelle façon est-il possible d'améliorer les capacités d'attention et de concentration au volant ? »; score variant entre 0-13). Le score total de l'IOC varie de 0 à 41, un score élevé représentant un intérêt, une ouverture et des connaissances supérieures. Pour faciliter les comparaisons, le score total et des trois sections est rapporté en pourcentage. Le second questionnaire, le CSC, fait ressortir les perceptions des conducteurs âgés quant aux changements survenus ou non pour 11 capacités (Tableau 5). Enfin, l'USC contient 20 questions portant sur les stratégies compensatoires répondues à l'aide d'échelles de Likert à 4 niveaux (Tableau 7). Les scores du CSC et de l'USC sont calculés respectivement en pourcentage et en fréquence et ce, pour chacune des 11 capacités ou des 20 stratégies compensatoires. L'IOC, le CSC et l'USC présentent une bonne cohérence interne (alphas de Cronbach : 0,54 à $0,90)$. Lors du post-test, un questionnaire sur les données sociodémographiques a aussi été complété afin de décrire les caractéristiques des participants. Chaque participant a été rencontré individuellement pendant environ 60 minutes en deux temps à $T_{0}$ et $T_{1}$ [8 à 10 semaines après l'intervention, laps de temps représentant un compromis pour favoriser l'indépendance entre les deux évaluations (diminuer le biais de rappel), et identifier l'impact de l'intervention] dans un local du Centre de recherche sur le vieillissement (CdRV). Pour explorer l'efficacité de l'OSCAR, le test $t$ bilatéral pour échantillons appariés a permis de comparer les résultats de l'IOC entre les deux temps de mesure. Les résultats du 
CSC et de l'USC ont été décrits à l'aide de fréquences et de pourcentages. Toutes les analyses statistiques ont été effectuées avec le logiciel SPSS (version 18.0). Cette recherche a reçu l'approbation du Comité d'éthique de la recherche du Centre de santé et de services sociaux de l'Institut universitaire de gériatrie de Sherbrooke.

\section{Résultats}

La recension des écrits a permis d'identifier 18 outils de dépistage ou de sensibilisation à la conduite automobile en lien avec le vieillissement disponibles dans les écrits scientifiques et sur des sites Internet d'organismes dédiés à la sécurité routière. Ces outils ont principalement été développés aux États-Unis dans les années 1990. Lors d'un premier forum d'informateurs clés, puisque ces outils variaient considérablement en termes de qualité et de contenu [21], les experts étaient d'avis qu'il était impossible de sélectionner un seul outil et que l'évaluation devait porter sur le contenu spécifique des outils. Une extraction des 110 items et des 142 capsules éducatives contenus dans les outils a donc été réalisée, suivie d'une analyse de leur contenu. En lien avec le modèle de Michon, les conseils des capsules éducatives portaient principalement sur le niveau stratégique, soit les objectifs de conduite et le contexte général, et sur le niveau tactique, c'est-à-dire les comportements impliqués dans l'application des règles de la route et les demandes immédiates de la route et du trafic incluant la performance des manœuvres. Les conseils du niveau stratégique les plus fréquents étaient (nombre d'outils) : éviter de conduire la nuit (16), consulter un professionnel de la santé (16), considérer les violations du Code de la route comme un signe précurseur (15), considérer les conditions météorologiques et le trafic (14) et utiliser la médication de façon sécuritaire (13), tandis que les conseils du niveau tactique étaient : utiliser les aides techniques et les équipements de l'automobile, par exemple les miroirs, de façon appropriée (11) et être capable d'analyser et de réagir rapidement (14). Une évaluation des items et des capsules éducatives a ensuite été réalisée par les experts menant à une première sélection. Ainsi, 24 des 110 items et 30 des 142 capsules éducatives ont été retenus pour un deuxième forum composé d'informateurs clés (Figure 1). À la suite de la deuxième rencontre, 15 items et 15 capsules éducatives en lien avec le vieillissement et la conduite automobile ont été sélectionnés et inclus dans une première version de l'Outil de sensibilisation des conducteurs âgés aux capacités requises ainsi qu'aux stratégies compensatoires pour une conduite automobile sécuritaire et responsable (OSCAR ; Tableau 1). Ainsi, l'intervention renvoie à plusieurs ressources incluant des stratégies compensatoires, des cours de perfectionnement et des ressources d'évaluation. Plusieurs assises théoriques ont par ailleurs guidé le développement d'OSCAR, soit les modèles : (i) Processus de production du handicap [22] du fonctionnement humain, (ii) Transthéorique de Prochaska [23], descriptif de l'adoption de comportements, et (iii) Michon [19], explicatif du traitement de l'information en conduite automobile. Ces modèles sont respectivement issus des domaines de la réadaptation, de la promotion de la santé et de la conduite automobile.

Lors de la première expérimentation, les participants ont évalué l'outil comme étant globalement «très utile » ou « utile », notamment pour se rappeler du lien entre le bilan routier et les capacités à conduire. Cette validation a permis de confirmer qu'étant concis, complet, clair, précis et rédigé dans un langage accessible, l'OSCAR favoriserait une prise de conscience, et permettrait aux aînés d'identifier les changements qui surviennent en eux, et de mieux connaître les stratégies compensatoires et les ressources disponibles. Entre autres, les participants rapportent que l'OSCAR pourrait être recommandé à certaines personnes de leur entourage ou utilisé pour parler de leurs préoccupations avec leurs proches ou un professionnel de la santé.

Lors de la deuxième expérimentation, les participants étaient âgés entre 67 et 84 ans, la majorité avait plus de 11 ans de scolarité et leurs revenus étaient supérieurs à 25000 dollars Canadien (Tableau 2). La plupart était retraité, vivait avec leur conjoint, percevait leur santé comme étant bonne, et réalisait leurs activités quotidiennes sans aide. La majorité conduisait tous les jours et deux des participants ont rapporté avoir déjà reçu une intervention similaire, soit le Drivers 55 Plus [24]. Aucun abandon n'est survenu entre les deux temps de mesure.

Les résultats démontrent que l'OSCAR a un impact positif sur l'intérêt, l'ouverture et les connaissances des conducteurs âgés globalement, ainsi que sur deux des trois dimensions ( $\mathrm{p}<0,01$; Tableau 3). Spécifiquement, les conducteurs se sont améliorés pour les dimensions «Intérêt et ouverture » et "Connaissances sur la sécurité routière et les capacités requises ». Malgré l'aspect exploratoire des comparaisons pour les dimensions, une tendance, mais pas de différence statistiquement significative, a été observée pour les "Connaissances sur l'impact $\mathrm{du}$ vieillissement sur les capacités et sur les stratégies compensatoires ».

La majorité $(85,4 \%)$ des participants a rapporté que la lecture de l'OSCAR leur avait permis de constater des changements reliés à l'âge qui sont survenus dans au moins une de leurs capacités, et plus du quart ont mentionné en avoir observé dans plus de huit capacités (Tableau 4). De plus, pour plus de la moitié des participants $(54,2 \%)$, l'OSCAR leur a permis d'identifier de confirmer l'apparition d'au moins un changement dans leurs capacités. Moins du quart $(22,9 \%)$ des participants a rapporté aucun 
Tableau 1 Quelques exemples de questions et de capsules éducatives se retrouvant dans l'Outil de Sensibilisation des aînés aux capacités requises et aux stratégies compensatoires pour une Conduite Automobile Sécuritaire et Responsable (OSCAR)

\section{Exemples de capacités ciblées Exemples de questions favorisant une réflexion sur les capacités}

1. Votre vision

Avez-vous plus de difficulté qu'avant à lire les panneaux de signalisation?

2. Votre jugement, temps de réaction et concentration

\section{Votre force et flexibilité}

4. Votre bilan routier et vos habitudes au volant

5. Votre médication et consommation d'alcool
Avez-vous plus de difficulté qu'avant à joindre la circulation sur une autoroute achalandée ?

Avez-vous plus de difficulté qu'avant à utiliser les pédales?

Vous est-il arrivé de ne pas savoir ce qu'un panneau de signalisation veut dire?

Prenez-vous de l'alcool?

\section{Exemples de capsules éducatives favorisant l'adoption de stratégies compensatoires}

Puisque la majorité de l'information reçue par un conducteur est de nature visuelle, une conduite sécuritaire nécessite d'avoir une bonne vision. S'ils sont détectés tôt, la plupart des problèmes visuels peuvent être traités efficacement. Faites évaluer votre vision par un spécialiste de la vue à chaque année.

Avec l'âge, la capacité à percevoir les profondeurs diminue. Il devient ainsi plus difficile de juger de la vitesse et de la distance des autres véhicules. Si vous vous sentez nerveux ou insécure lorsque vous devez joindre la circulation sur une autoroute achalandée, éviter ces situations. S'il est difficile pour vous de conduire sur les autoroutes achalandées, évitez d'y circuler aux heures de pointe. Suivre un cours de perfectionnement pourrait aussi vous aider à améliorer vos habiletés de conduite dans ces situations. Lors du freinage, il est nécessaire d'avoir une force suffisante aux jambes. Cette force permet aussi de maintenir une stabilité dans le contrôle des pédales sans ressentir de fatigue. Faites régulièrement de l'exercice physique pour renforcer et assouplir les muscles de vos jambes. Une réadaptation pourrait aussi être requise pour améliorer votre force et votre flexibilité.

Informez-vous auprès d'un professionnel de la santé.

Les routes, la signalisation et les lois changent continuellement. Un cours de perfectionnement peut vous aider à mettre à jour vos connaissances sur la conduite automobile. Ce cours peut aussi vous aider à vous ajuster aux limites occasionnées par le vieillissement et à améliorer vos habiletés de conduite.

Il est bien connu que, pour les personnes de tout âge, l'alcool détériore considérablement les habiletés de conduite. Toutefois, puisque la tolérance à l'alcool diminue avec l'âge, il est important d'éviter de consommer des boissons alcoolisées avant de prendre le volant. De plus, la consommation d'alcool augmente les effets nuisibles des médicaments sur vos habiletés de conduite. Si vous avez bu, ne conduisez pas.

* La version complète de l'outil est disponible sur demande en contactant l'auteure principale. 
Tableau 2 Caractéristiques des participants $(n=48)$

Moyenne \pm É.T.

Âge (ans)

Nombre d'années depuis l'obtention du permis de conduire

Variables catégoriques

Genre

Femme

Variables socioéconomiques Statut civil

Célibataire

$74,7 \pm 7,4$

$24,4 \pm 11,2$

Fréquence (\%)

$24(50,0)$

$2(4,2)$

Veuf (veuve)

$9(18,8)$

Marié(e)/en couple

$30(62,5)$

Divorcé

$7(14,6)$

Situation de vie

Demeure avec le conjoint

$30(62,5)$

Demeure avec un enfant

$3(6,3)$

$15(31,3)$

Scolarité (années)

Seul

$17(35,4)$

$11(22,9)$

$20(41,7)$

Revenus (\$ Canadien)

$12-14$

$\geq 14$

$\leq 25000$

$9(18,8)$

25 001-40 000

$19(39,6)$

$>40000$

Occupation

Emploi à temps complet

$20(41,7)$

0

Emploi à temps partiel

$5(10,5)$

Retraité

$43(89,5)$

$1(2,1)$

Santé et fonctionnement Santé - présentement

Plutôt mauvaise

$27(56,3)$

Plutôt bonne

$20(41,7)$

$13(27,1)$

Santé - comparaison avec il y a 5 ans

Vraiment bonne

$31(64,6)$

$4(8,3)$

Santé - comparaison avec personnes du même

Pareille

$1(2,1)$ âge

Mieux

Pire

$21(43,8)$

$26(54,2)$

Activitiés de la vie quotidienne réalisées sans aide

Pareille

$47(97,9)$

Utilisation du téléphone

$46(95,8)$

Épicerie, magasinage

$44(91,7)$

$38(79,2)$

Entretien ménager

$45(93,8)$

Prise de médication

$46(95,8)$

Habitudes de conduite

Exposition à la conduite ( $\mathrm{km} / \mathrm{semaine})$

1-50

$13(27,1)$

$11(22,9)$

$8(16,7)$

$8(16,7)$

$8(16,7)$

Fréquence de conduite (jours/semaine)

101-150

$4(8,3)$

$17(35,4)$

$27(56,3)$ 


\begin{tabular}{|c|c|c|c|c|c|}
\hline \multirow[t]{2}{*}{ Dimensions de l'IOC (\# de questions ; score) } & & \multicolumn{3}{|c|}{ Statistiques descriptives } & \multirow{2}{*}{$\begin{array}{l}\text { Comparaison } \\
\text { des moyennes }\end{array}$} \\
\hline & & \% (É.T.) & Min & $\operatorname{Max}$ & \\
\hline \multirow[t]{2}{*}{ Intérêt et ouverture $(5 ; / 19)$} & $\mathrm{T}_{0}$ & $47,6(20,5)$ & 10,5 & 89,5 & $<0,01^{*}$ \\
\hline & $\mathrm{T}_{1}$ & $55,0(18,8)$ & 15,8 & 94,7 & \\
\hline \multirow{2}{*}{$\begin{array}{l}\text { Connaissances - sécurité routière et les capacités } \\
\text { requises }(9 ; / 9)\end{array}$} & $\mathrm{T}_{0}$ & $84,0(10,5)$ & 55,6 & 100 & $<0,01^{*}$ \\
\hline & $\mathrm{T}_{1}$ & $87,4(10,4)$ & 55,6 & 100 & \\
\hline \multirow{2}{*}{$\begin{array}{l}\text { Connaissances - impact du vieillissement sur } \\
\text { capacités + stratégies compensatoires }(13 ; / 13)\end{array}$} & $\mathrm{T}_{0}$ & $78,4(17,0)$ & 30,8 & 100 & 0,74 \\
\hline & $\mathrm{T}_{1}$ & $79,0(19,6)$ & 30,8 & 100 & \\
\hline \multirow[t]{2}{*}{ Total } & $\mathrm{T}_{0}$ & $65,3(12,2)$ & 39,0 & 90,2 & $<0,01 *$ \\
\hline & $\mathrm{T}_{1}$ & $69,8(12,0)$ & 43,9 & 92,7 & \\
\hline
\end{tabular}

Tableau 4 Résultats globaux aux changements survenus dans les capacités (CSC; T1;n=48)

Nombre de capacités Types de changement

$\begin{array}{lll}\text { Oui, je l'ai constaté grâce à } & \begin{array}{l}\text { Oui, je le savais et l'outil me l'a } \\ \text { confirmé Fréquence }(\%)\end{array} & \begin{array}{l}\text { Non, aucun changement pour } \\ \text { ces capacités Fréquence }(\%)\end{array}\end{array}$

\begin{tabular}{lrrr}
\hline 0 & $7(14,6)$ & $22(45,8)$ & $11(22,9)$ \\
$1-4$ & $16(33,3)$ & $20(41,7)$ & $17(35,4)$ \\
$5-8$ & $12(25,0)$ & $6(12,5)$ & $9(18,8)$ \\
$9-11$ & $13(27,1)$ & 0 & $11(22,9)$
\end{tabular}

changement dans neuf capacités ou plus (Tableau 4). Plus spécifiquement, « Rester attentif sur la route quand vous êtes préoccupé » est la capacité où le plus $(27,1 \%)$ de participants ont observé des changements (Tableau 5). Pour la majorité des participants, l'OSCAR a permis d'identifier des changements dans les six capacités suivantes : « Rester attentif sur la route quand vous êtes préoccupé », «Bien voir ce qui se passe sur les côtés », « Freiner rapidement en situation d'imprévus », « Bien voir de loin », « Réagir rapidement », et « $\grave{A}$ garder une vitesse stable sans ressentir de fatigue ». Enfin, « Rester attentif sur la route quand vous ressentez de la douleur » est la seule capacité où la majorité des participants n'ont noté aucun changement (Tableau 5).

Après avoir pris connaissance de l'OSCAR, la moitié des participants ont rapporté utiliser plus de cinq stratégies compensatoires ou avoir l'intention d'utiliser entre une et 10 stratégies (Tableau 6). De plus, la majorité $(52,1 \%$ ) a mentionné utiliser déjà plus de 10 stratégies. La plupart $(87,5 \%)$ des conducteurs âgés ont rapporté avoir au moins une stratégie qu'il ne prévoit pas utiliser (Tableau 6). Plus spécifiquement, les trois stratégies les plus utilisées par la majorité des participants à la suite de l'OSCAR sont : « Laisser une plus grande distance entre votre véhicule et celui devant » $(45,8 \%)$, «Éviter les heures de pointe » $(43,8 \%)$ et « Être plus conscient de ce qui se passe tout autour de vous » $(43,8 \%$; Tableau 7$)$. «Planifier l'achat d'une voiture à transmission automatique » est la stratégie utilisée par la majorité $(70,8 \%)$ des participants avant l'OSCAR (Tableau 7). «Conduire à la limite de vitesse permise » est la stratégie que la plupart $(68,8 \%)$ des participants avaient l'intention d'utiliser, et moins d'un cinquième $(16,7 \%)$ planifiait utiliser d'autres stratégies. Enfin, «Installer sur votre véhicule des miroirs spéciaux » (68,8 \%), «Éviter les autoroutes achalandées » $(41,7 \%)$ et « Choisir des routes qui requièrent peu de changements de voie » $(37,5 \%)$ étaient les trois stratégies que la majorité des participants rapportaient ne pas avoir l'intention d'utiliser (Tableau 7). 


\begin{tabular}{|c|c|c|c|}
\hline \multirow{2}{*}{$\begin{array}{l}\text { Est-ce que l'OSCAR vous a permis de } \\
\text { constater ou de confirmer l'apparition de } \\
\text { changements au niveau de votre capacité à... }\end{array}$} & \multicolumn{3}{|l|}{ Types de changement } \\
\hline & $\begin{array}{l}\text { Oui, je l'ai constaté } \\
\text { grâce à l'OSCAR } \\
\text { Fréquence }(\%)\end{array}$ & $\begin{array}{l}\text { Oui, je le savais et } \\
\text { l'outil me l'a confirmé } \\
\text { Fréquence }(\%)\end{array}$ & $\begin{array}{l}\text { Non, aucun changement } \\
\text { pour ces capacités } \\
\text { Fréquence }(\%)\end{array}$ \\
\hline Bien voir de loin & $3(6,3)$ & $24(50,0)$ & $21(43,8)$ \\
\hline Bien voir ce qui se passe sur les côtés & $2(4,2)$ & $27(56,3)$ & $19(39,6)$ \\
\hline $\begin{array}{l}\text { Juger de la vitesse et de la distance des autres } \\
\text { véhicules }\end{array}$ & $9(18,8)$ & $21(43,8)$ & $18(37,5)$ \\
\hline Réagir rapidement & $6(12,5)$ & $24(50,0)$ & $18(37,5)$ \\
\hline $\begin{array}{l}\text { Traiter les informations provenant de différentes } \\
\text { sources }\end{array}$ & $7(14,6)$ & $20(41,7)$ & $21(43,8)$ \\
\hline $\begin{array}{l}\text { Rester attentif sur la route quand vous êtes } \\
\text { préoccupé }\end{array}$ & $6(12,5)$ & $27(56,3)$ & $15(31,3)$ \\
\hline Rester attentif sur la route quand vous êtes fatigué & $13(27,1)$ & $22(45,8)$ & $13(27,1)$ \\
\hline $\begin{array}{l}\text { Rester attentif sur la route quand vous ressentez } \\
\text { de la douleur }\end{array}$ & $10(20,8)$ & $12(25,0)$ & $26(54,2)$ \\
\hline Regarder par-dessus votre épaule & $8(16,7)$ & $22(45,8)$ & $18(37,5)$ \\
\hline Freiner rapidement en situation d'imprévus & $3(6,3)$ & $25(52,1)$ & $20(41,7)$ \\
\hline $\begin{array}{l}\text { À garder une vitesse stable sans ressentir de } \\
\text { fatigue }\end{array}$ & $5(10,4)$ & $24(50,0)$ & $19(39,6)$ \\
\hline
\end{tabular}

\begin{tabular}{|lcccc}
\hline Tableau 6 Résultats globaux de l'utilisation des stratégies compensatoires (USC $\left.; \mathrm{T}_{1} ; \mathrm{n}=48\right)$ \\
\hline Nombre de stratégies & Types of changement & & \\
\cline { 2 - 5 } & Oui Fréquence $(\%)$ & $\begin{array}{c}\text { Non, je le faisais déjà } \\
\text { avant Fréquence }(\%)\end{array}$ & $\begin{array}{c}\text { Non, mais je prévois le } \\
\text { faire Fréquence }(\%)\end{array}$ & $\begin{array}{c}\text { Non et je ne prévois pas } \\
\text { le faire Fréquence }(\%)\end{array}$ \\
\hline & $11(22,9)$ & $1(2,1)$ & $24(50,0)$ & $6(12,5)$ \\
$1-5$ & $13(27,1)$ & $10(20,8)$ & $23(47,9)$ & $33(68,7)$ \\
$6-10$ & $14(29,2)$ & $12(25,0)$ & $1(2,1)$ & $9(18,8)$ \\
$11-15$ & $10(20,8)$ & $16(33,3)$ & 0 & 0 \\
$16-20$ & 0 & $9(18,8)$ & 0 & 0
\end{tabular}

\section{Discussion}

Développé de façon rigoureuse [25] et validé auprès de 48 ainés, la présente étude a permis d'obtenir l'OSCAR, un outil d'intervention visant à promouvoir et maintenir la mobilité des personnes âgées via une conduite automobile sécuritaire et responsable. Les forces et les limites de l'OSCAR peuvent être comparées aux outils que l'on retrouve dans les écrits scientifiques et la littérature grise. Comme la majorité de ces outils, l'OSCAR a l'avantage de s'appuyer sur une ou plusieurs bases théoriques solides, d'être facile à comprendre et à compléter, de requérir un court temps pour être lu, et de contenir des sections pouvant être utilisées séparément. Contrairement à quelques rares outils, l'OSCAR n'est actuellement disponible pas en anglais, et en format papier et électronique. Néanmoins, l'OSCAR est parmi les rares outils qui ont été développés grâce à une synthèse rigoureuse des données probantes [21], deux consultations d'experts, une validation auprès de 12 aînés [25] et d'une validation auprès de 48 autres aînés. Par ailleurs, l'OSCAR amène directement les aînés à se questionner sur les changements qui sont susceptibles d'être survenus à la suite du vieillissement et qui ont été démontrés comme étant reliés à une conduite automobile sécuritaire. 


\begin{tabular}{|c|c|c|c|c|}
\hline \multirow{2}{*}{$\begin{array}{l}\text { Depuis la lecture de l'outil (OSCAR) } \\
\text { lors de la première rencontre, } \\
\text { avez-vous commencé à... }\end{array}$} & \multicolumn{4}{|l|}{ Types de changement } \\
\hline & Oui Fréquence (\%) & $\begin{array}{l}\text { Non, je le faisais } \\
\text { déjà avant } \\
\text { Fréquence }(\%)\end{array}$ & $\begin{array}{l}\text { Non, mais je } \\
\text { prévois le faire } \\
\text { Fréquence }(\%)\end{array}$ & $\begin{array}{l}\text { Non et je ne } \\
\text { prévois pas le faire } \\
\text { Fréquence }(\%)\end{array}$ \\
\hline Éviter de conduire à la noirceur & $5(10,4)$ & $25(52,1)$ & $4(8,3)$ & $14(29,2)$ \\
\hline Être plus vigilant aux intersections & $20(41,7)$ & $26(54,2)$ & $2(4,2)$ & 0 \\
\hline $\begin{array}{l}\text { Regarder à droite de la chaussée plutôt } \\
\text { que les phares des voitures venant en } \\
\text { sens inverse }\end{array}$ & $14(29,2)$ & $26(54,2)$ & $4(8,3)$ & $4(8,3)$ \\
\hline Éviter les virages à gauche & $12(25,0)$ & $16(33,3)$ & $5(10,4)$ & $15(31,3)$ \\
\hline Éviter les autoroutes achalandées & $12(25,0)$ & $13(27,1)$ & $3(6,3)$ & $20(41,7)$ \\
\hline Éviter les heures de pointe & $21(43,8)$ & $16(33,3)$ & $1(2,1)$ & $10(20,8)$ \\
\hline $\begin{array}{l}\text { Laisser une plus grande distance entre } \\
\text { votre véhicule et celui devant }\end{array}$ & $22(45,8)$ & $25(52,1)$ & $1(2,1)$ & 0 \\
\hline $\begin{array}{l}\text { Etre plus conscient de ce qui se passe } \\
\text { tout autour de vous }\end{array}$ & $21(43,8)$ & $27(56,3)$ & 0 & 0 \\
\hline Faire de l'exercice physique & $15(31,3)$ & $28(58,3)$ & $4(8,3)$ & $1(2,1)$ \\
\hline $\begin{array}{l}\text { Surveiller la circulation, les panneaux de } \\
\text { signalisation, les feux de circulation, les } \\
\text { cyclistes et les piétons à l'approche des } \\
\text { intersections }\end{array}$ & $15(31,3)$ & $32(66,7)$ & 0 & $1(2,1)$ \\
\hline Planifier votre trajet à l'avance & $14(29,2)$ & $32(66,7)$ & $2(4,2)$ & 0 \\
\hline $\begin{array}{l}\text { Planifier l'achat d'une voiture à } \\
\text { transmission automatique }\end{array}$ & $7(14,6)$ & $34(70,8)$ & 0 & $7(14,6)$ \\
\hline $\begin{array}{l}\text { Éviter de conduire lors de conditions } \\
\text { pouvant interférer avec votre habileté à } \\
\text { vous concentrer }\end{array}$ & $15(31,3)$ & $26(54,2)$ & $6(12,5)$ & $1(2,1)$ \\
\hline Réduire les distractions au volant & $15(31,3)$ & $30(62,5)$ & $3(6,3)$ & 0 \\
\hline $\begin{array}{l}\text { Installer sur votre véhicule des miroirs } \\
\text { spéciaux }\end{array}$ & $1(2,1)$ & $6(12,5)$ & $8(16,7)$ & $33(68,8)$ \\
\hline $\begin{array}{l}\text { Choisir des routes qui requièrent peu de } \\
\text { changements de voie }\end{array}$ & $9(18,8)$ & $18(37,5)$ & $3(6,3)$ & $18(37,5)$ \\
\hline $\begin{array}{l}\text { Suivre un programme d'exercices pour } \\
\text { améliorer votre force et votre flexibilité }\end{array}$ & $9(18,8)$ & $22(45,8)$ & $8(16,7)$ & $9(18,8)$ \\
\hline Conduire à la limite de vitesse permise & $14(29,2)$ & $3(6,3)$ & $33(68,8)$ & $1(2,1)$ \\
\hline $\begin{array}{l}\text { Vous questionner sur la cause des } \\
\text { réactions négatives des autres } \\
\text { automobilistes à votre égard }\end{array}$ & $19(39,6)$ & $24(50,0)$ & $1(2,1)$ & $4(8,3)$ \\
\hline $\begin{array}{l}\text { Être à l'écoute des commentaires de votre } \\
\text { entourage concernant votre conduite }\end{array}$ & $20(41,7)$ & $25(52,1)$ & $2(4,2)$ & $1(2,1)$ \\
\hline
\end{tabular}

Enfin, les changements pouvant être survenus sont associés plus clairement aux habitudes à modifier et aux stratégies à utiliser, incluant les ressources disponibles.

Globalement, les conducteurs âgés ont rapporté être intéressés par l'OSCAR, qui permet d'augmenter leur intérêt, leur ouverture et leurs connaissances. À la suite de la lecture de l'OSCAR, la majorité des participants ont aussi confirmé avoir constaté que des changements s'étaient produits dans au moins une de leurs capacités et la moitié rapportent avoir commencé à utiliser plus de cinq stratégies compensatoires. Ces résultats sont similaires à ceux d'autres études qui ont démontré les 
bénéfices d'interventions éducationnelles sur la conduite automobile. Par exemple, lors de l'expérimentation de l'étude d'Owsley et collègues [26], les conducteurs dans le groupe d'intervention éducationnelle ont rapporté conduire significativement moins de jours, à moins d'endroits et moins de fois par semaine comparés à ceux n'ayant pas participé à l'intervention. Même si l'OSCAR a permis d'augmenter l'intérêt, l'ouverture et les connaissances des conducteurs âgés, les changements observés étaient modestes. L'exposition à l'OSCAR ayant toutefois été minimale, c'est-à-dire que les conducteurs âgés étaient invités à le lire une seule fois, mais ne pouvaient pas le conserver, ces résultats pourraient suggérer que l'intervention doit être répétée et personnalisée. En fait, une étude a démontré que les programmes intensifs étaient plus efficaces [27]. Par ailleurs, le score initial élevé pour l'intérêt, l'ouverture et les connaissances des aînés peut aussi expliquer les changements modestes observés à la suite de l'OSCAR.

Des études antérieures ont démontré que certains conducteurs âgés manquent de prise de conscience quant à l'effet du vieillissement et de leurs problèmes de santé sur leurs capacités de conduite. Une récente étude réalisée auprès de 307 conducteurs âgés de 65 à 96 ans a trouvé aucune association entre la performance objective et la confiance perçue [28]. Une autre étude a démontré qu'une performance inférieure à un test de conduite n'était pas associée à l'évitement général de situations de conduite difficiles [27]. Par ailleurs, plusieurs conducteurs âgés ont besoin d'aide pour adopter de nouveaux comportements tel que changer une habitude de conduite ou utiliser une stratégie compensatoire. En fait, une récente étude a démontré que l'autorégulation est un processus complexe dont les motivations varient considérablement d'une situation à l'autre, et sont similaires aux habitudes de vie ou aux préférences [29]. Enfin, une autre étude a démontré que les conducteurs âgés ayant une atteinte de leurs fonctions cognitives pourraient ne pas s'autoréguler ou participer d'eux-mêmes à un programme sur la conduite auto [30]. Une étude a néanmoins démontré que les perceptions des conducteurs âgés sont associées avec leur pratique actuelle d'autorégulation [31].

Considérant la complexité des changements de comportement [23], l'OSCAR favoriserait une prise de conscience qui est nécessaire, mais non suffisante au changement. En effet, à la suite d'une première lecture de l'OSCAR, il est peu probable que les conducteurs âgés apportent des changements dans leur façon de conduire, même si la majorité des aînés ont rapporté que l'outil était utile pour confirmer les changements survenus. D'ailleurs, bien que la majorité des aînés ayant consulté l'OSCAR affirment que l'outil leur avait permis de confirmer des changements au niveau de leurs capacités, la majorité mentionne aussi ne pas prévoir modifier leur façon de conduire. Ainsi, il est attendu que la plupart des aînés auront besoin de soutien, soit d'un proche ou d'un professionnel de la santé, pour modifier leurs habitudes de conduite. Par ailleurs, certains aînés ne peuvent être sensibilisés puisqu'ils ont une perception altérée, c'està-dire une diminution de l'autocritique envers les effets de leur vieillissement et de leurs problèmes de santé sur leurs capacités au volant. Il importe ainsi d'adapter l'OSCAR aux proches et aux intervenants afin de mieux les outiller à accompagner les conducteurs âgés dans le changement de leurs habitudes de conduite et l'utilisation de stratégies compensatoires. La majorité des aînés indiquaient d'ailleurs que l'OSCAR leur serait utile pour discuter de leurs préoccupations reliées à leur conduite automobile avec un professionnel de la santé, un moniteur de conduite ou leurs proches. À notre connaissance, cinq outils «Au volant de ma santé » [32], How to help an older driver [33], Elderly drivers: Is your loved one driving safely? [34], We need to talk-Family conversations with older drivers [35] et « Les conducteurs âgés au Canada et leur famille » [36], ont été développés ou incluent une section pour les membres de la famille.

Enfin, l'aide d'un moniteur de conduite ou d'un professionnel de la santé peut aussi être requise. Selon McNamara et collègues [37], les aînés auraient plus tendance à abandonner leur permis de conduite à cause de leur âge avancé, d'une confiance diminuée en ses capacités de conduite et de conseils de cesser de conduire de leur médecin de famille. Les suggestions d'autres individus pour limiter ou arrêter de conduire pourraient toutefois influencer davantage les conducteurs âgés que leur autoévaluation de leurs capacités de conduite [38]. Par exemple, une étude a démontré que les conducteurs qui ont reçu une intervention d'éducation étaient plus enclins à reconnaître que leur vision était moins bonne, et à rapporter éviter plus fréquemment des situations de conduite difficiles et à s'autoréguler davantage [26]. Tout comme pour les familles, il existe des interventions pour assister les professionnels de la santé avec les défis associés aux conducteurs âgés : Promising approaches for enhancing elderly mobility [39], Driving transitions education. Tools, scripts, and practice exercises [40] et « Les conducteurs âgés au Canada : Professionnels de la santé » [36].

Cette étude a permis le développement et une première validation de l'OSCAR, une intervention de sensibilisation aux capacités requises et aux stratégies compensatoires pour une conduite automobile sécuritaire et responsable. Malgré son caractère exploratoire, cette étude a aussi des limites. D'abord, elle a été réalisée avec un échantillon de convenance, ce qui peut probablement avoir un impact positif sur l'intérêt, l'ouverture et les connaissances des participants envers la conduite auto. Dans les études subséquentes, il importe d'examiner l'impact de l'OSCAR en présence d'un groupe contrôle et sur la conduite sur route. De plus, avant d'être implanté à grande échelle dans 
la communauté, l'impact de l'OSCAR sur la prévention des accidents et des blessures sur route doit être démontré. Enfin, il est important de mieux comprendre comment les habitudes de conduite se modifient à travers le temps et les facteurs qui influencent ces changements.

\section{Conclusion}

La présente étude a permis de développer un Outil d'intervention afin de promouvoir et maintenir la mobilité des personnes âgées via une conduite automobile sécuritaire. L'Outil de sensibilisation des conducteurs âgés aux capacités requises ainsi qu'aux stratégies compensatoires pour une conduite automobile sécuritaire et responsable (OSCAR) comprend une série de 15 questions et 15 capsules éducatives en lien avec le vieillissement et la conduite automobile. Selon les conducteurs âgés ayant participé à la présente étude, l'OSCAR est un outil rédigé dans un langage accessible, qui favorise une prise de conscience envers les capacités requises pour une conduite automobile sécuritaire. L'outil permettrait aux conducteurs âgés d'identifier les changements qui surviennent en eux et les habitudes à modifier, incluant l'utilisation de stratégies compensatoires et les ressources disponibles telles que les cours de perfectionnement et les centres d'évaluation. D'autres études doivent être réalisées afin d'adapter l'OSCAR aux proches et aux intervenants afin de mieux les outiller à soutenir les conducteurs âgés dans le changement de leurs habitudes de conduite et l'utilisation de stratégies compensatoires. Des modifications pourraient être apportées à l'OSCAR afin que, par exemple, les recommandations aident plus directement les aînés à utiliser les stratégies compensatoires. Enfin, les facilitateurs et les obstacles aux changements des habitudes de conduite doivent être explorés, et les besoins et les attitudes des aînés quant à l'utilisation de ces stratégies doivent être mieux compris et intégrés à l'OSCAR. Tout en favorisant la prévention des accidents et des blessures sur le réseau routier, l'OSCAR pourrait ultimement permettre aux aînés de maintenir ou d'augmenter leur mobilité dans la communauté et leur participation sociale.

Remerciements Cette étude a été financée par les Instituts de recherche en santé du Canada, l'Université de Sherbrooke et le Centre de recherche sur le vieillissement du Centre de santé et de services sociaux de l'Institut Universitaire de gériatrie de Sherbrooke. Merci à Mesdames Francisca Beloin et Marie-Michèle Bédard, stagiaires de recherche, pour leur travail respectivement de recension et de collecte de données, qui a contribué à la faisabilité de cette étude. Monia D'Amours, agente de recherche, a contribué significativement à la coordination du projet, ainsi qu'à la collecte et l'analyse des données. Enfin, merci aux conducteurs âgés qui ont accepté de donner de leur temps pour participer à l'étude.

\section{Références}

1. Classen $\mathrm{S}$ et al. (2010) Item development and validity testing for a self- and proxy report : the safe driving behavior measure. American Journal of Occupational Therapy 64(2): 296-305.

2. Wang C et al. (2009) Physician's guide to assessing and counseling older drivers. Washington, DC : National Highway Traffic Safety Administration.

3. Myers AM et al. (2008) Process evaluation of the american automobile association roadwise review CD-ROM : Observed and reported experiences of older drivers. Topics in Geriatric Rehabilitation 24(3): 224-238.

4. Audet T et al. (2007) Conduite automobile. In Arcand M. R. (ed) Précis pratique de gériatrie, $3^{\mathrm{e}}$ édition, Edisem inc. 1099-112.

5. Eberhard JW et al. (2006) Strategies and tools to enable safe mobility for older adults. Topics in Geriatric Rehabilitation 22(1): $3-17$

6. Hutcherson DG (1989) Self-monitoring of driving for the elderly : Evidence for use of a driving diary. Physical and Occupational Therapy in Geriatrics 7(1-2): 171-201.

7. Pellerito JMJ (2005) Driver rehabilitation and community mobility: principles and practice, Elsevier Mosby.

8. Gauthier J (2008) Interventions relatives à l'utilisation d'un véhicule routier : guide de l'ergothérapeute. Ordre des ergothérapeutes du Québec.

9. Association canadienne des ergothérapeutes ACE (2009) Plan d'action national pour la prévention des blessures chez les conducteurs âgés. Ottawa ON : CAOT Publications ACE.

10. Eby DW et al. (2003) Improving older driver knowledge and self-awareness through self-assessment : the driving decisions workbook. J Safety Res 34(4): 371-81.

11. Kiernan B et al (1999) Improving driving performance of senior drivers through self-monitoring with a driving diary. Physical and Occupational Therapy in Geriatrics 16(1-2): 55-64.

12. Eby DW (1998) The assessment of older drivers' capabilities: a review of the literature.

13. Hunt L \& Arbesman M (2008) Evidence-Based and Occupational Perspective of Effective Interventions for Older Clients That Remediate or Support Improved Driving Performance. American Journal of Occupational Therapy 62(2): 136-48.

14. OMS ACSP (1986) Santé et bien-être social Canada. Charte d'Ottawa pour la promotion de la santé. Ottawa (Ontario) Canada.

15. Filiatrault J (2009) Introduction à la santé publique. Présentation de l'unité de santé publique en réadaptation, École de réadaptation-programme d'ergothérapie et de physiothérapie, Université de Sherbrooke.

16. Crepeau EB et al. (2009) Willard \& Spackman's Occupational Therapy, 11th ed.: Lippincott William.

17. Bouchard S. \& Cyr S. (2005) Recherche psychosociale : pour harmoniser recherche et pratique. Presses de l'Université du Québec.

18. Streiner DL \& Norman GR (2008) Health measurment scales: A practical guide to their development and use, OU Press, Editor.

19. Michon J (1985) A critical view of driver behavior models: what do we know, what should we do? in Human behavior and traffic safety, L.S. Evans, R.C, ed., New York: Plenum Press, p. 485-520.

20. Machin D et al. (2009) Sample-size tables for clinical studies (3rd ed.), West sussex, UK : Oxford : Wiley-Blackwell. 
21. Levasseur M et al. (2011) Tools raising older drivers' awareness of capabilities needed to drive safely: a scoping study. Canadian Occupational Therapy Conference 2011. Saskatoon, Saskatchewan, June 15-18, 2011.

22. Fougeyrollas P et al. (1998) Classification québécoise Processus de production $d u$ handicap, Québec : Réseau international sur le Processus de production du handicap (RIPPH)/ Société canadienne pour la Classification internationale des déficiences, incapacités et handicaps (SCCIDIH). p. 166.

23. Prochaska J \& Velicer W (1997) The transtheoretical model of health behavior change. American Journal of Health Promotion 12(1): $38-48$.

24. American Automobile Association (1994) Drivers 55 Plus: Self-rating form. Foundation for Traffic Safety (available at: http://www.aaafoundation.org/drivers-65?button=driver55, accessed August 8, 2013.).

25. Levasseur M (2012) Outil de sensibilisation aux capacités requises pour une conduite automobile sécuritaire et responsable. Émission Au cour du Monde : La conduite des personnes âgées. Radio-Canada. 7 juin, 2012.

26. Owsley C, Stalvey BT \& Phillips JM (2003) The efficacy of an educational intervention in promoting selfregulation among high-risk older drivers. Accid Anal Prev 35(3): 393-400.

27. Jones V et al. (2012) The effect of a low and high resource intervention on older drivers' knowledge, behaviors and risky driving. Accid Anal Prev 49: 486-92.

28. Horswill MS et al. (2011) Older drivers' insight into their hazard perception ability. Accid Anal Prev 43(6): 2121-7.

29. Molnar LJ et al. (2013) Driving avoidance by older adults: is it always self-regulation? Accid Anal Prev 57: 96-104.

30. Wong IY, Smith SS \& Sullivan KA (2012) The relationship between cognitive ability, insight and self-regulatory behaviors: findings from the older driver population. Accid Anal Prev 49: 316-21.

31. Blanchard RA \& Myers AM (2010) Examination of driving comfort and self-regulatory practices in older adults using in-vehicle devices to assess natural driving patterns. Accid Anal Prev 42(4): 1213-9.
32. Société de l'assurance automobile du Québec (2011) Health in the driver's seat. Available at : http://www.saaq.gouv.qc.ca/publications/prevention/health_driver. pdf Accessed July 29, 2013.

33. American Automobile Association and Foundation for traffic safety (2000), How to Help an Older Driver: A Guide for Planning Safe Transportation. Washington, DC. Available at : http://www.aaafoundation.org/sites/default/files/ODlarge.pdf Accessed August 1st 2013.

34. Senior solutions of America (2007) Elderly Drivers : Is your loved one driving safely? Washington, DC. Available at : http://elderlydrivingassessments.com/images/individ/elderdrivers familychecklist.pdf Accessed August 1st 2013.

35. Sedgwick County Department on Aging (2010) We Need to Talk - Family Conversations with Older Drivers. available at : http://hartfordauto.thehartford.com/UI/Downloads/FamConHtd. pdf accessed August 14, 2013.

36. Association canadienne des ergothérapeutes (2009) Les conducteurs âgés au Canada et leur famille. http://www.securitedesconducteursages.ca/consommateur/index. html site web consulté le 18 février 2014.

37. McNamara A et al. (2013) What factors influence older people in the decision to relinquish their driver's licence? A discrete choice experiment. Accident Analysis \& Prevention 55: 178-184.

38. Ross L A et al. (2012) Self-rated driving and driving safety in older adults. Accid Anal Prev 48: 523-7.

39. Molnar LJ, Eby DW, \& Miller LL (2003) Promising Approaches for Enhancing Elderly Mobility. University of Michigan Transportation Research Institute. Ann Arbor, MI. available at : http://deepblue.lib.umich.edu/bitstream/handle/2027.42/60921/? sequence $=1$ Accessed October 23, 2014.

40. American Society on Aging and the National Highway Traffic Safety Administration (2009) Driving Transitions Education. Tools, Scripts, and Practice Exercises. Available at: http://www.google.ca/url?sa =t\&rct=j\&q=\&esrc=s\&source $=$ web $\& c d=1 \& v e d=0 C B 8 Q F j A A \& u r l=h t t p \% 3 \mathrm{~A} \% 2 \mathrm{~F} \% 2 \mathrm{Fwww}$.nhtsa. gov\%2FDOT\%2FNHTSA\%2FTraffic \%2520Injury \%2520Control \%2FArticles\%2FAssociated\%2520Files\%2F811152.pdf\&ei=P2 NJVNifAY6ayATy3oL4AQ\&usg=AFQjCNGH7UBfq79RgZgft_ mSTZhG9MkD6Q\&sig2=pBfKwRMp3CCT98wzHoIgjA\& bvm $=$ bv.77880786,d.aWw Accessed October 23, 2014. 